\title{
Infectious crystalline keratopathy
}

\author{
C B JAMES, P J McDONNELL, AND M G FALCON \\ From the Eye Department, St Thomas's Hospital, London SE1 7EH
}

SUMmARY We present a patient who developed a crystalline keratopathy after a penetrating keratoplasty. This rare complication can be caused by bacterial infection, and the patient responded to the appropriate antibiotic therapy. The literature is reviewed and possible causes and mechanisms of the crystalline appearance are discussed.

A crystalline appearance within the stroma of the cornea may be caused by a variety of conditions including lipid and metabolic disorders such as cystinosis and monoclonal gammopathy in association with multiple myeloma. More unexpectedly it can occur as a complication of penetrating keratoplasty following either infection ${ }^{1-5}$ or rejection. ${ }^{67} \mathrm{We}$ report here on a patient who developed a crystalline keratopathy following a graft. The cause of the keratopathy was considered to be infective and the condition resolved on antibacterial treatment.

\section{Case reports}

A 32-year-old man with advanced keratoconus underwent an uneventful penetrating keratoplasty in December 1984. A whole donor eye was used, and the graft was sutured in place with a continuous $10 / 0$ nylon suture. The surgery was uncomplicated though technically difficult because. of thinning of the host cornea peripherally. Six days later there was an aequeous leak, with hypotony. The anterior chamber was shallow, the suture loose, and the graft oedematous. Following placement of a single 10/0 nylon suture at the site of the leak the anterior chamber reformed and the leak was sealed; the cornea cleared, and there were no synechiae. Fifteen days later the continuous suture had loosened slightly between 8 and 10 o'clock at the site of the leak, and mucus had become adherent to it. This caused no problems, and no change in management was required save for the addition of $10 \%$ acetylcystine drops four times daily.

Ten months after surgery, in October 1985, the suture had loosened superiorly, and this section was removed. At this stage the corrected visual acuity

Correspondence to M G Falcon, FRCS. was $6 / 5$, and the patient was taking prednisolone (Predsol) twice daily in addition to the acetylcystine. A few days after the removal of the suture the graft developed a superficial corneal crystalline stromal infiltrate (Fig. 1) at 9 o'clock, which appeared to be associated with a suture track. The epithelium was intact and the remainder of the graft clear; the anterior chamber contained occasional cells. Conjunctival swabs were taken, but no bacteria grew on blood agar plates. A corneal scraping was not carried out because of the intact epithelium.

Treatment with prednisolone was continued and intensive topical gentamicin and methicillin started. After five days, when the possibility of a streptoccocal infection was realised in the light of the experience of others, ${ }^{1-5}$ treatment was changed to topical penicillin and corticosteroids. The lesion

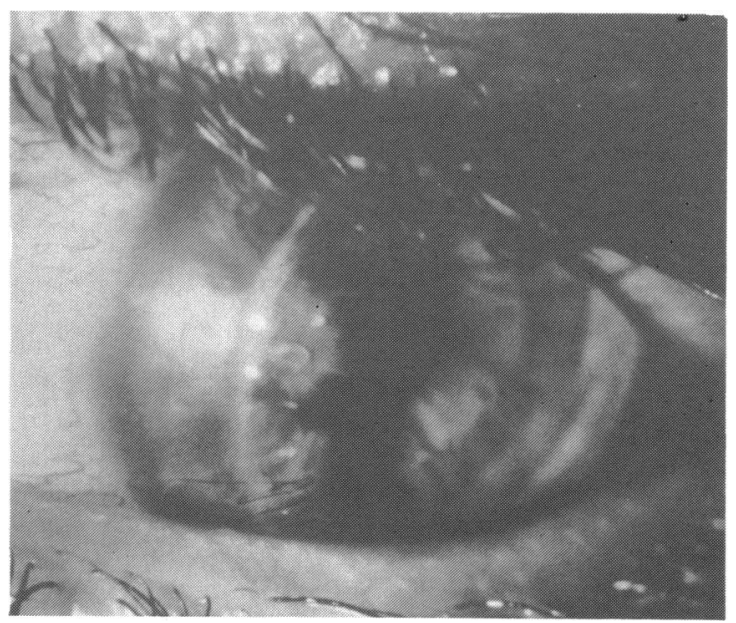

Fig. 1 The initial appearance of the crystalline lesion in October 1985. 


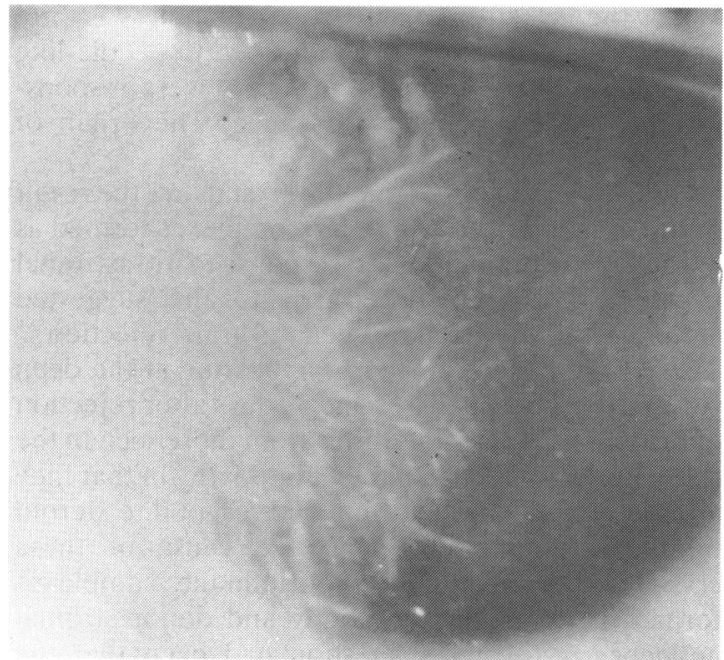

Fig. 2 The development of further crystalline deposits in February 1986.

regressed. After six weeks, in November 1985, the antibiotics were stopped.

In February 1986, three months after withdrawal of the antibiotics, the stromal lesion recurred and was associated with a crystalline opacity spreading into the graft at midstromal level. Topical penicillin and steroid was again started. A corneal scrape failed to grow any organisms when directly plated on to blood agar; a Gram stain was not performed. The eye initially worsened. It became painful, with the development of photophobia and redness, but the clinical signs were unchanged (Figs. 2, 3). Penicillin

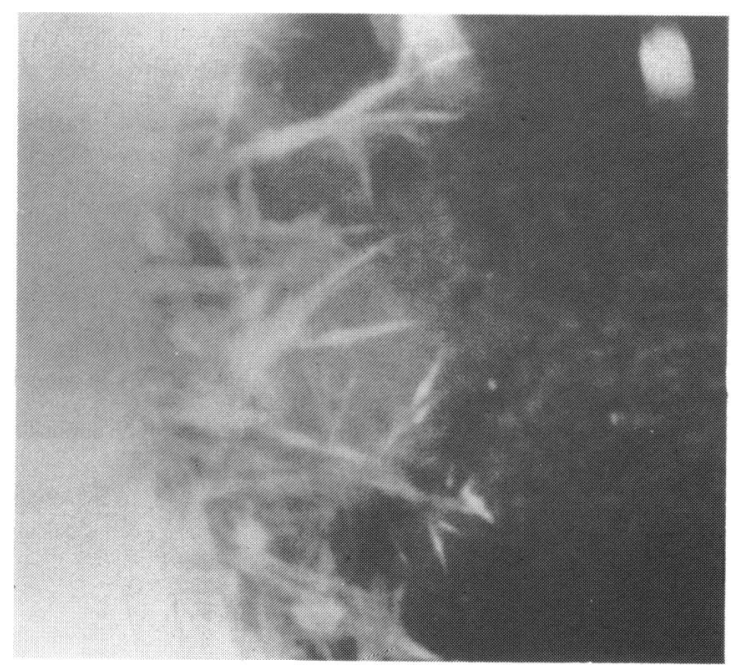

Fig. 3 Magnified view of the crystalline deposits in February 1986. was stopped, and, because of the possibility of a more serious infection, the patient was given a subconjunctival injection of gentamicin and methicillin and was started on systemic treatment with the same antibiotics. The eye improved on this regimen, with a reduction in the area of opacification and no progression of the crystalline keratopathy. Two weeks after the acute episode the patient was using gentamicin and methicillin every two hours and prednisolone three times daily. The gentamicin was stopped one week later.

Three months later the crystals had markedly regressed. The patient at this time was taking prednisolone and methicillin twice daily, having received antibiotics for a total of three months. At follow-up in September 1986 there had been no recurrence, and the corrected visual acuity was $6 / 9$. Slight stromal scarring persisted peripherally at 9 o'clock, but all crystalline appearances had regressed completely. Subsequent follow-up revealed no change (Fig. 4).

\section{Discussion}

Gorovoy et al. ${ }^{3}$ reported the first case of a crystal-like deposition associated with infection in a corneal graft. In his case, as in other reports, there was no sign of ocular inflammation. The crystalline appearance progressed over five months, and the patient eventually required a regraft. There is no mention of any culture being performed, and no antibacterial therapy was given. Analysis of the corneal button removed showed a localised ingrowth of epithelium associated with a suture, and from this area colonies of Gram-positive bacteria were seen extending between the stromal lamellae.

Meisler et al.' presented an initial series of three

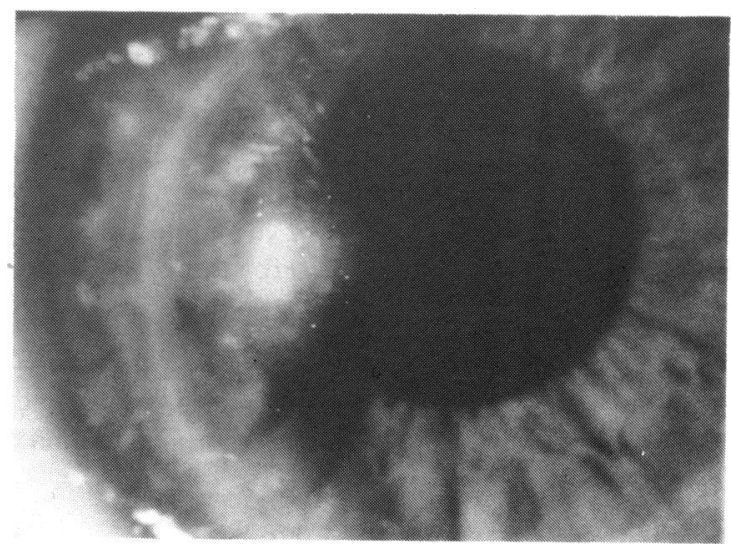

Fig. 4 The corneal appearance in February 1987. Note that central white area is light reflection. 
patients with infectious crystalline keratopathy: two developed after penetrating keratoplasty, one in a herpetic eye. Two of the three patients grew an $\alpha$-haemolytic streptococcus either from a corneal scrape or from a diagnostic keratectomy. All three of his patients were treated with antibiotics. The lesions resolved, leaving a vạiable amount of scarring or corneal oedema.

Samples et al. ${ }^{2}$ reported on a patient, again showing no sign of ocular inflammation, who was treated for a presumed fungal infection. Diagnostic keratectomy had failed to demonstrate any organisms. The situation worsened so that a further graft had to be performed. Examination of the corneal button showed dense bacterial colonies beneath Bowman's membrane.

Reiss ${ }^{5}$ reported on a patient in whom Streptococcus viridans had been cultured from a corneal scrape. A repeated graft had to be performed, however, and microscopy of the button again demonstrated the presence of intrastromal bacteria.

The case reported in this paper follows a similar pattern to those reviewed above. The recurrence of the lesion after a short course of antibiotics indicates that topical treatment must be continued for some months to eradicate the infection. The location of the organism within the stroma, demonstrated by electron microscopy ${ }^{1-35}$ not only makes it difficult to obtain specimens to culture an organism but also presumably lends protection to the bacteria from the effect of topically applied antibiotic.

The way in which the bacteria penetrate the corneal stroma and the reason for their growth in the anterior part of the stroma is open to speculation. A suture track would seem the most probable mode of bacterial entry into the stroma, but any break in Bowman's membrane, no matter how caused, would suffice. The immunosuppressive effect of topical corticosteroids may also play a part. All but one of the cases reported have occurred in patients with corneal grafts, for whom prolonged steroid treatment is usually required. There have been no reports of a similar process occurring in cataract surgery, where the period of steroid treatment is much shorter. Samples et al. ${ }^{2}$ suggested that the predilection of the organism for the anterior stroma reflects an advantageous environment for growth at this depth in the cornea.

The nature of the crystalline appearance itself is also obscure. Reiss et al. . $^{5}$ suggested that it may be formed by the bacterial colonies themselves as they grow between the lamellae of the corneal stroma.
With the electron microscope Samples et al. ${ }^{2}$ observed electron dense bodies with needle-like projections and considered that they were responsible for the crystalline appearance. The origin of these bodies was uncertain.

It might be suggested that the crystals are the result of the precipitation of immune complexes formed as a result of an immunological response to intrastromal bacteria. This would be akin to the suggested aetiology of the crystals seen in graft rejection. ${ }^{67}$ These have been reported as appearing at the deep stromal level between five and 30 days after rejection of the graft. The crystals differ from those seen in the case of infectious crystalline keratopathy in that they are found in the deep stroma and respond to steroid treatment alone. The suggested cause of these crystals is a precipitation of immune complexes formed between host antibody and donor stromal antigens. A further suggestion had been that the crystals may be the result of lipid deposition. ${ }^{2}$ The appearance is not, however, typical of that seen in lipid keratopathy and it would not be expected to resolve with antibiotic treatment.

\section{CONCLUSION}

Stromal infection of a corneal graft may present as a superficial or midstromal crystalline keratopathy in a quiet or minimally inflamed eye. It is associated with the presence of intrastromal bacteria and must be differentiated from the deep crystalline keratopathy seen in graft rejection. Treatment should be with thorough, prolonged application of antibiotics, which is curative.

\section{References}

1 Meisler DM, Roger HL, Langston MD, Naab TJ, Aaby AA, McMahon TJ. Infectious crystalline keratopathy. Am J Ophthalmol 1984; 97: 337-44.

2 Samples JR, Baumgartner SD, Binder PS. Infectious crystalline keratopathy: an electron microscope analysis. Cornea 1985; 4: 118-26.

3 Gorovoy MS, Stern GA, Hood CI, Allen C. Intrastromal noninflamatory bacterial colonization of a corneal graft. Arch Ophthalmol 1983; 101: 1749-52.

4 Meisler DM, Roger HS, Langstrom MD, Aaby AA, Stern GA, Binder PS. Infectious corneal crystalline formation. Invest Ophthalmol Vis Sci 1984; 25 (suppl): 23.

5 Reiss GR, Campbell RJ, Bourne WM. Infectious crystalline keratopathy. Surv Ophthalmol 1986; 31: 69-72.

6 Mason CM, Sugar A, Meyer RF. Intrastromal crystalline deposits following corneal graft rejection. Cornea 1984; 3: 89-94.

7 Girard LJ. Crystalline deposits and graft rejections. Cornea 1985; 4: 67 .

Accepted for publication 4 June 1987. 